\title{
Effects of Hydrogen Bonding on the Acidity of Adenine, Guanine and Their 8-oxo Derivatives
}

\author{
Tom L. McConnell, Craig A. Wheaton, Ken C. Hunter and Stacey D. Wetmore* \\ Department of Chemistry, Mount Allison University, 63C York Street, \\ Sackville, New Brunswick, E4L 1G8, Canada
}

Supporting Information

(Tables $\mathrm{S} 1$ - S5, 33 pages) 
Table S1: Calculated (N9) Acidities $\left(\mathrm{kJ} \mathrm{mol}^{-1}\right)$ of Adenine Complexes with Water, Ammonia and Hydrogen Fluoride $^{\mathrm{a}}$

\begin{tabular}{|c|c|c|c|c|c|c|c|}
\hline N1(N6) & N7(N6) & N7(C8) & N1(C2) & acidity & $\Delta(\text { acidity })^{b}$ & additive $^{\mathrm{c}}$ & $\Delta^{\mathrm{d}}$ \\
\hline $\mathrm{H}_{2} \mathrm{O}$ & & & & 1388.5 & 18.3 & & \\
\hline $\mathrm{HF}$ & & & & 1365.2 & 41.6 & & \\
\hline \multirow[t]{6}{*}{$\mathrm{NH}_{3}$} & & & & 1406.9 & -0.1 & & \\
\hline & $\mathrm{H}_{2} \mathrm{O}$ & & & 1381.8 & 25.0 & & \\
\hline & $\mathrm{HF}$ & & & 1348.7 & 58.1 & & \\
\hline & $\mathrm{NH}_{3}$ & & & 1403.3 & 3.5 & & \\
\hline & & $\mathrm{NH}_{3}$ & & 1394.1 & 12.7 & & \\
\hline & & & $\mathrm{NH}_{3}$ & 1395.3 & 11.5 & & \\
\hline $\mathrm{H}_{2} \mathrm{O}$ & $\mathrm{H}_{2} \mathrm{O}$ & & & 1365.0 & 41.8 & 43.3 & -1.5 \\
\hline $\mathrm{H}_{2} \mathrm{O}$ & $\mathrm{HF}$ & & & 1334.0 & 72.8 & 76.4 & -3.6 \\
\hline $\mathrm{H}_{2} \mathrm{O}$ & $\mathrm{NH}_{3}$ & & & 1385.8 & 21.0 & 21.8 & -0.8 \\
\hline $\mathrm{HF}$ & $\mathrm{H}_{2} \mathrm{O}$ & & & 1343.2 & 63.6 & 66.6 & -3.0 \\
\hline $\mathrm{HF}$ & $\mathrm{HF}$ & & & 1312.7 & 94.1 & 99.7 & -5.6 \\
\hline $\mathrm{HF}$ & $\mathrm{NH}_{3}$ & & & 1363.8 & 43.0 & 45.1 & -2.1 \\
\hline $\mathrm{NH}_{3}$ & $\mathrm{H}_{2} \mathrm{O}$ & & & 1382.2 & 24.6 & 24.9 & -0.3 \\
\hline
\end{tabular}




\begin{tabular}{|c|c|c|c|c|c|c|c|}
\hline $\mathrm{NH}_{3}$ & $\mathrm{HF}$ & & & 1352.4 & 54.4 & 58.0 & -3.6 \\
\hline $\mathrm{NH}_{3}$ & $\mathrm{NH}_{3}$ & & & 1401.9 & 4.9 & 3.4 & 1.5 \\
\hline $\mathrm{H}_{2} \mathrm{O}$ & & $\mathrm{NH}_{3}$ & & 1377.7 & 29.1 & 31.0 & -1.9 \\
\hline $\mathrm{HF}$ & & $\mathrm{NH}_{3}$ & & 1356.0 & 50.8 & 54.3 & -3.5 \\
\hline $\mathrm{NH}_{3}$ & & $\mathrm{NH}_{3}$ & & 1395.1 & 11.7 & 12.6 & -0.9 \\
\hline & $\mathrm{H}_{2} \mathrm{O}$ & & $\mathrm{NH}_{3}$ & 1371.5 & 35.3 & 36.5 & -1.2 \\
\hline & $\mathrm{HF}$ & & $\mathrm{NH}_{3}$ & 1338.9 & 67.9 & 69.6 & -1.7 \\
\hline & $\mathrm{NH}_{3}$ & & $\mathrm{NH}_{3}$ & 1392.4 & 14.4 & 15.0 & -0.6 \\
\hline & & $\mathrm{NH}_{3}$ & $\mathrm{NH}_{3}$ & 1383.2 & 23.6 & 24.2 & -0.6 \\
\hline
\end{tabular}

${ }^{\mathrm{a} B} 3 \mathrm{LYP} / 6-311+\mathrm{G}(2 \mathrm{~d}, \mathrm{p})$ single-point calculations on $\mathrm{B} 3 \mathrm{LYP} / 6-31+\mathrm{G}(\mathrm{d}, \mathrm{p})$ geometries. See Figure 1 for structure, atomic numbering and notation for adenine and its complexes. ${ }^{\mathrm{b}}$ The calculated (N9) acidities of isolated adenine $\left(1406.8 \mathrm{~kJ} \mathrm{~mol}^{-1}\right)$ minus the calculated acidity of the adenine-derivative complex. A positive value represents an increase in the acidity. ${ }^{c}$ The sum of the effects of the individual hydrogen bonds on the acidity. ${ }^{\mathrm{d}}$ The difference between the additive and the calculated effect of two molecules, where a positive value indicates that the effects are greater than additive. 
Table S2: Calculated (N9) Acidities $\left(\mathrm{kJ} \mathrm{mol}^{-1}\right)$ of Oxoadenine in Complexes with Water, Ammonia and Hydrogen Fluoride ${ }^{\mathrm{a}}$

\begin{tabular}{|c|c|c|c|c|c|}
\hline N1(N6) & O8(N7) & acidity & $\Delta{\text { (acidity })^{b}}^{b}$ & additive $^{\mathrm{c}}$ & $\Delta^{\mathrm{d}}$ \\
\hline $\mathrm{H}_{2} \mathrm{O}$ & & 1392.3 & 15.8 & & \\
\hline $\mathrm{HF}$ & & 1368.2 & 39.9 & & \\
\hline $\mathrm{NH}_{3}$ & & 1411.1 & -3.0 & & \\
\hline & $\mathrm{H}_{2} \mathrm{O}$ & 1391.7 & 16.4 & & \\
\hline & $\mathrm{HF}$ & 1359.4 & 48.7 & & \\
\hline & $\mathrm{NH}_{3}$ & 1414.8 & -6.7 & & \\
\hline $\mathrm{H}_{2} \mathrm{O}$ & $\mathrm{H}_{2} \mathrm{O}$ & 1377.5 & 30.6 & 32.2 & -1.6 \\
\hline $\mathrm{H}_{2} \mathrm{O}$ & $\mathrm{HF}$ & 1346.7 & 61.4 & 64.5 & -3.1 \\
\hline $\mathrm{H}_{2} \mathrm{O}$ & $\mathrm{NH}_{3}$ & 1399.9 & 8.2 & 9.1 & -0.9 \\
\hline $\mathrm{HF}$ & $\mathrm{H}_{2} \mathrm{O}$ & 1355.9 & 52.2 & 56.3 & -4.1 \\
\hline $\mathrm{HF}$ & $\mathrm{HF}$ & 1324.9 & 83.2 & 88.6 & -5.4 \\
\hline $\mathrm{HF}$ & $\mathrm{NH}_{3}$ & 1378.6 & 29.5 & 33.2 & -3.7 \\
\hline $\mathrm{NH}_{3}$ & $\mathrm{H}_{2} \mathrm{O}$ & 1395.1 & 13.0 & 13.4 & -0.4 \\
\hline $\mathrm{NH}_{3}$ & $\mathrm{HF}$ & 1365.0 & 43.1 & 45.7 & -2.6 \\
\hline $\mathrm{NH}_{3}$ & $\mathrm{NH}_{3}$ & 1417.2 & -9.1 & -9.7 & 0.6 \\
\hline
\end{tabular}


${ }^{\mathrm{a}} \mathrm{B} 3 \mathrm{LYP} / 6-311+\mathrm{G}(2 \mathrm{~d}, \mathrm{p})$ single-point calculations on B3LYP/6-31+G(d,p) geometries. See Figure 1 for structure, atomic numbering and notation for 8-oxoadenine and its complexes. ${ }^{\mathrm{b}}$ The calculated (N9) acidities of isolated 8-oxoadenine $\left(1408.1 \mathrm{~kJ} \mathrm{~mol}^{-1}\right)$ minus the calculated acidity of the 8-oxoadenine complex. A positive value represents an increase in the acidity. ${ }^{\mathrm{c}}$ The sum of the effects of the individual hydrogen bonds on the acidity. ${ }^{\mathrm{d}}$ The difference between the additive and the calculated effect of two molecules, where a positive value indicates that the effects are greater than additive. 
Table S3: Calculated (N9) Acidities $\left(\mathrm{kJ} \mathrm{mol}^{-1}\right)$ of Guanine in Complexes with Water, Ammonia and Hydrogen Fluoride ${ }^{\mathrm{a}}$

\begin{tabular}{|c|c|c|c|c|c|c|}
\hline O6-N7 & N3(N2) & O6(N1) & acidity & $\Delta(\text { acidity })^{b}$ & additive $^{c}$ & $\Delta^{\mathrm{d}}$ \\
\hline $\mathrm{H}_{2} \mathrm{O}$ & & & 1370.6 & 33.0 & & \\
\hline $\mathrm{HF}$ & & & 1353.7 & 49.9 & & \\
\hline $\mathrm{NH}_{3}$ & & & 1385.9 & 17.7 & & \\
\hline & $\mathrm{H}_{2} \mathrm{O}$ & & 1382.2 & 21.4 & & \\
\hline & $\mathrm{HF}$ & & 1364.5 & 39.1 & & \\
\hline & $\mathrm{NH}_{3}$ & & 1401.0 & 2.5 & & \\
\hline & & $\mathrm{H}_{2} \mathrm{O}$ & 1393.9 & 9.7 & & \\
\hline & & $\mathrm{HF}$ & 1368.5 & 35.1 & & \\
\hline & & $\mathrm{NH}_{3}$ & 1412.6 & -9.1 & & \\
\hline $\mathrm{H}_{2} \mathrm{O}$ & $\mathrm{H}_{2} \mathrm{O}$ & & 1350.8 & 52.8 & 54.4 & -1.6 \\
\hline $\mathrm{H}_{2} \mathrm{O}$ & $\mathrm{HF}$ & & 1334.3 & 69.3 & 72.1 & -2.8 \\
\hline $\mathrm{H}_{2} \mathrm{O}$ & $\mathrm{NH}_{3}$ & & 1370.1 & 33.5 & 35.5 & -2.0 \\
\hline $\mathrm{HF}$ & $\mathrm{H}_{2} \mathrm{O}$ & & 1334.6 & 69.0 & 71.3 & -2.3 \\
\hline $\mathrm{HF}$ & $\mathrm{HF}$ & & 1317.4 & 86.2 & 89.0 & -2.8 \\
\hline $\mathrm{HF}$ & $\mathrm{NH}_{3}$ & & 1353.6 & 50.0 & 52.4 & -2.4 \\
\hline
\end{tabular}




\begin{tabular}{|c|c|c|c|c|c|c|}
\hline $\mathrm{NH}_{3}$ & $\mathrm{H}_{2} \mathrm{O}$ & & 1365.5 & 38.1 & 39.1 & -1.0 \\
\hline $\mathrm{NH}_{3}$ & $\mathrm{HF}$ & & 1348.1 & 55.5 & 56.8 & -1.3 \\
\hline $\mathrm{NH}_{3}$ & $\mathrm{NH}_{3}$ & & 1384.3 & 19.3 & 20.2 & -0.9 \\
\hline $\mathrm{H}_{2} \mathrm{O}$ & & $\mathrm{H}_{2} \mathrm{O}$ & 1364.0 & 39.5 & 42.7 & -3.2 \\
\hline $\mathrm{H}_{2} \mathrm{O}$ & & $\mathrm{HF}$ & 1339.5 & 64.1 & 68.1 & -4.0 \\
\hline $\mathrm{H}_{2} \mathrm{O}$ & & $\mathrm{NH}_{3}$ & 1381.9 & 21.7 & 23.9 & -2.2 \\
\hline $\mathrm{HF}$ & & $\mathrm{H}_{2} \mathrm{O}$ & 1347.2 & 56.4 & 59.6 & -3.2 \\
\hline $\mathrm{HF}$ & & $\mathrm{HF}$ & 1323.0 & 80.6 & 85.0 & -4.4 \\
\hline $\mathrm{HF}$ & & $\mathrm{NH}_{3}$ & 1365.0 & 38.6 & 40.8 & -2.2 \\
\hline $\mathrm{NH}_{3}$ & & $\mathrm{H}_{2} \mathrm{O}$ & 1377.6 & 26.0 & 27.4 & -1.4 \\
\hline $\mathrm{NH}_{3}$ & & $\mathrm{HF}$ & 1352.7 & 50.8 & 52.8 & -2.0 \\
\hline $\mathrm{NH}_{3}$ & & $\mathrm{NH}_{3}$ & 1396.0 & 7.6 & 8.6 & -1.0 \\
\hline & $\mathrm{H}_{2} \mathrm{O}$ & $\mathrm{H}_{2} \mathrm{O}$ & 1373.8 & 29.8 & 31.1 & -1.3 \\
\hline & $\mathrm{H}_{2} \mathrm{O}$ & $\mathrm{HF}$ & 1349.6 & 53.9 & 56.5 & -2.6 \\
\hline & $\mathrm{H}_{2} \mathrm{O}$ & $\mathrm{NH}_{3}$ & 1392.3 & 11.3 & 12.3 & -1.0 \\
\hline & $\mathrm{HF}$ & $\mathrm{H}_{2} \mathrm{O}$ & 1357.7 & 45.9 & 48.8 & -2.9 \\
\hline & $\mathrm{HF}$ & $\mathrm{HF}$ & 1332.9 & 70.7 & 74.2 & -3.5 \\
\hline & $\mathrm{HF}$ & $\mathrm{NH}_{3}$ & 1376.4 & 27.2 & 30.0 & -2.8 \\
\hline
\end{tabular}




\begin{tabular}{|c|c|c|c|c|c|c|}
\hline & $\mathrm{NH}_{3}$ & $\mathrm{H}_{2} \mathrm{O}$ & 1391.7 & 11.9 & 12.2 & -0.3 \\
\hline & $\mathrm{NH}_{3}$ & $\mathrm{HF}$ & 1368.8 & 34.8 & 37.6 & -2.8 \\
\hline & $\mathrm{NH}_{3}$ & $\mathrm{NH}_{3}$ & 1409.2 & -5.6 & -6.6 & 1.0 \\
\hline $\mathrm{H}_{2} \mathrm{O}$ & $\mathrm{H}_{2} \mathrm{O}$ & $\mathrm{H}_{2} \mathrm{O}$ & 1362.7 & 40.8 & 64.1 & -23.3 \\
\hline $\mathrm{H}_{2} \mathrm{O}$ & $\mathrm{H}_{2} \mathrm{O}$ & $\mathrm{HF}$ & 1322.3 & 81.3 & 89.5 & -8.2 \\
\hline $\mathrm{H}_{2} \mathrm{O}$ & $\mathrm{H}_{2} \mathrm{O}$ & $\mathrm{NH}_{3}$ & 1362.7 & 40.8 & 45.3 & -4.5 \\
\hline $\mathrm{H}_{2} \mathrm{O}$ & $\mathrm{HF}$ & $\mathrm{H}_{2} \mathrm{O}$ & 1329.2 & 74.4 & 81.8 & -7.4 \\
\hline $\mathrm{H}_{2} \mathrm{O}$ & $\mathrm{HF}$ & $\mathrm{HF}$ & 1305.2 & 98.4 & 107.2 & -8.8 \\
\hline $\mathrm{H}_{2} \mathrm{O}$ & $\mathrm{HF}$ & $\mathrm{NH}_{3}$ & 1347.4 & 56.2 & 63.0 & -6.8 \\
\hline $\mathrm{H}_{2} \mathrm{O}$ & $\mathrm{NH}_{3}$ & $\mathrm{H}_{2} \mathrm{O}$ & 1363.2 & 40.4 & 45.2 & -4.8 \\
\hline $\mathrm{H}_{2} \mathrm{O}$ & $\mathrm{NH}_{3}$ & $\mathrm{HF}$ & 1341.5 & 62.1 & 70.6 & -8.5 \\
\hline $\mathrm{H}_{2} \mathrm{O}$ & $\mathrm{NH}_{3}$ & $\mathrm{NH}_{3}$ & 1380.2 & 23.3 & 26.4 & -3.1 \\
\hline $\mathrm{HF}$ & $\mathrm{H}_{2} \mathrm{O}$ & $\mathrm{H}_{2} \mathrm{O}$ & 1328.9 & 74.7 & 81.0 & -6.3 \\
\hline $\mathrm{HF}$ & $\mathrm{H}_{2} \mathrm{O}$ & $\mathrm{HF}$ & 1306.0 & 97.5 & 106.4 & -8.9 \\
\hline $\mathrm{HF}$ & $\mathrm{H}_{2} \mathrm{O}$ & $\mathrm{NH}_{3}$ & 1346.1 & 57.5 & 62.2 & -4.7 \\
\hline $\mathrm{HF}$ & $\mathrm{HF}$ & $\mathrm{H}_{2} \mathrm{O}$ & 1313.2 & 90.4 & 98.7 & -8.3 \\
\hline $\mathrm{HF}$ & $\mathrm{HF}$ & $\mathrm{HF}$ & 1289.0 & 114.6 & 124.1 & -9.5 \\
\hline $\mathrm{HF}$ & $\mathrm{HF}$ & $\mathrm{NH}_{3}$ & 1331.1 & 72.5 & 79.9 & -7.4 \\
\hline
\end{tabular}




\begin{tabular}{|c|c|c|c|c|c|c|}
\hline $\mathrm{HF}$ & $\mathrm{NH}_{3}$ & $\mathrm{H}_{2} \mathrm{O}$ & 1346.8 & 56.8 & 62.1 & -5.3 \\
\hline $\mathrm{HF}$ & $\mathrm{NH}_{3}$ & $\mathrm{HF}$ & 1325.6 & 78.0 & 87.5 & -9.5 \\
\hline $\mathrm{HF}$ & $\mathrm{NH}_{3}$ & $\mathrm{NH}_{3}$ & 1363.3 & 40.3 & 43.3 & -3.0 \\
\hline $\mathrm{NH}_{3}$ & $\mathrm{H}_{2} \mathrm{O}$ & $\mathrm{H}_{2} \mathrm{O}$ & 1358.4 & 45.2 & 48.8 & -3.6 \\
\hline $\mathrm{NH}_{3}$ & $\mathrm{H}_{2} \mathrm{O}$ & $\mathrm{HF}$ & 1334.5 & 69.1 & 74.2 & -5.1 \\
\hline $\mathrm{NH}_{3}$ & $\mathrm{H}_{2} \mathrm{O}$ & $\mathrm{NH}_{3}$ & 1376.2 & 27.4 & 30.0 & -2.6 \\
\hline $\mathrm{NH}_{3}$ & $\mathrm{HF}$ & $\mathrm{H}_{2} \mathrm{O}$ & 1342.6 & 61.0 & 66.5 & -5.5 \\
\hline $\mathrm{NH}_{3}$ & $\mathrm{HF}$ & $\mathrm{HF}$ & 1318.1 & 85.5 & 91.9 & -6.4 \\
\hline $\mathrm{NH}_{3}$ & $\mathrm{HF}$ & $\mathrm{NH}_{3}$ & 1360.9 & 42.6 & 47.7 & -5.1 \\
\hline $\mathrm{NH}_{3}$ & $\mathrm{NH}_{3}$ & $\mathrm{H}_{2} \mathrm{O}$ & 1376.2 & 27.4 & 29.9 & -2.5 \\
\hline $\mathrm{NH}_{3}$ & $\mathrm{NH}_{3}$ & $\mathrm{HF}$ & 1353.8 & 49.8 & 55.3 & -5.5 \\
\hline $\mathrm{NH}_{3}$ & $\mathrm{NH}_{3}$ & $\mathrm{NH}_{3}$ & 1393.3 & 10.3 & 11.1 & -0.8 \\
\hline
\end{tabular}

${ }^{\mathrm{a} B} 3 \mathrm{LYP} / 6-311+\mathrm{G}(2 \mathrm{~d}, \mathrm{p})$ single-point calculations on B3LYP/6-31+G(d,p) geometries. See Figure 1 for structure, atomic numbering and notation for guanine and its complexes. ${ }^{\mathrm{b}}$ The calculated (N9) acidities of isolated guanine $\left(1403.6 \mathrm{~kJ} \mathrm{~mol}^{-1}\right)$ minus the calculated acidity of the guanine complex. A positive value represents an increase in the acidity. ${ }^{\mathrm{c}}$ The sum of the effects of the individual hydrogen bonds on the acidity. ${ }^{\mathrm{d}}$ The difference between the additive and the calculated effect of more than one molecule, where a positive value indicates that the effects are greater than additive. 
Table S4: Calculated (N1) Acidities $\left(\mathrm{kJ} \mathrm{mol}^{-1}\right)$ of Oxoguanine in Complexes with Water, Ammonia and Hydrogen Fluoride ${ }^{\mathrm{a}}$

\begin{tabular}{|c|c|c|c|c|c|c|c|}
\hline O6(N7) & O8(N7) & N3(N2) & O6(N1) & acidity & $\Delta{\text { (acidity })^{b}}^{b}$ & additive $^{\mathrm{c}}$ & $\Delta^{\mathrm{d}}$ \\
\hline $\mathrm{H}_{2} \mathrm{O}$ & & & & 1401.8 & 6.6 & & \\
\hline $\mathrm{HF}$ & & & & 1379.6 & 28.7 & & \\
\hline \multirow[t]{10}{*}{$\mathrm{NH}_{3}$} & & & & 1421.1 & -12.8 & & \\
\hline & $\mathrm{H}_{2} \mathrm{O}$ & & & 1389.1 & 19.2 & & \\
\hline & $\mathrm{HF}$ & & & 1360.1 & 48.3 & & \\
\hline & $\mathrm{NH}_{3}$ & & & 1412.9 & -4.6 & & \\
\hline & & $\mathrm{H}_{2} \mathrm{O}$ & & 1387.8 & 20.5 & & \\
\hline & & $\mathrm{HF}$ & & 1370.3 & 38.1 & & \\
\hline & & $\mathrm{NH}_{3}$ & & 1407.1 & 1.2 & & \\
\hline & & & $\mathrm{H}_{2} \mathrm{O}$ & 1398.1 & 10.3 & & \\
\hline & & & $\mathrm{HF}$ & 1371.1 & 37.2 & & \\
\hline & & & $\mathrm{NH}_{3}$ & 1417.5 & -9.1 & & \\
\hline $\mathrm{H}_{2} \mathrm{O}$ & $\mathrm{HF}$ & & & 1359.5 & 48.9 & 54.9 & -6.0 \\
\hline $\mathrm{HF}$ & $\mathrm{H}_{2} \mathrm{O}$ & & & 1356.4 & 51.9 & 47.9 & 4.0 \\
\hline $\mathrm{HF}$ & $\mathrm{HF}$ & & & 1336.5 & 71.8 & 77.0 & -5.2 \\
\hline
\end{tabular}




\begin{tabular}{|c|c|c|c|c|c|c|}
\hline $\mathrm{H}_{2} \mathrm{O}$ & $\mathrm{H}_{2} \mathrm{O}$ & & 1382.8 & 25.5 & 27.1 & -1.6 \\
\hline $\mathrm{H}_{2} \mathrm{O}$ & $\mathrm{HF}$ & & 1366.0 & 42.3 & 44.7 & -2.4 \\
\hline $\mathrm{H}_{2} \mathrm{O}$ & $\mathrm{NH}_{3}$ & & 1401.4 & 7.0 & 7.8 & -0.8 \\
\hline $\mathrm{HF}$ & $\mathrm{H}_{2} \mathrm{O}$ & & 1361.5 & 46.8 & 49.2 & -2.4 \\
\hline $\mathrm{HF}$ & $\mathrm{HF}$ & & 1343.5 & 64.9 & 66.8 & -1.9 \\
\hline $\mathrm{HF}$ & $\mathrm{NH}_{3}$ & & 1381.3 & 27.1 & 29.9 & -2.8 \\
\hline $\mathrm{NH}_{3}$ & $\mathrm{H}_{2} \mathrm{O}$ & & 1401.3 & 7.1 & 7.7 & -0.6 \\
\hline $\mathrm{NH}_{3}$ & $\mathrm{HF}$ & & 1385.0 & 23.4 & 25.3 & -1.9 \\
\hline $\mathrm{NH}_{3}$ & $\mathrm{NH}_{3}$ & & 1417.7 & -9.3 & -11.6 & 2.3 \\
\hline $\mathrm{H}_{2} \mathrm{O}$ & & $\mathrm{H}_{2} \mathrm{O}$ & 1393.6 & 14.7 & 16.9 & -2.2 \\
\hline $\mathrm{H}_{2} \mathrm{O}$ & & $\mathrm{HF}$ & 1370.5 & 37.8 & 43.8 & -6.0 \\
\hline $\mathrm{H}_{2} \mathrm{O}$ & & $\mathrm{NH}_{3}$ & 1411.3 & -2.9 & -2.5 & -0.4 \\
\hline $\mathrm{HF}$ & & $\mathrm{H}_{2} \mathrm{O}$ & 1375.1 & 33.2 & 39.0 & -5.8 \\
\hline $\mathrm{HF}$ & & $\mathrm{HF}$ & 1351.2 & 57.2 & 65.9 & -8.7 \\
\hline $\mathrm{HF}$ & & $\mathrm{NH}_{3}$ & 1392.8 & 15.6 & 19.6 & -4.0 \\
\hline $\mathrm{NH}_{3}$ & & $\mathrm{H}_{2} \mathrm{O}$ & 1411.7 & -3.4 & -2.5 & -0.9 \\
\hline $\mathrm{NH}_{3}$ & & $\mathrm{HF}$ & 1387.4 & 21.0 & 24.4 & -3.4 \\
\hline $\mathrm{NH}_{3}$ & & $\mathrm{NH}_{3}$ & 1429.3 & -21.0 & -21.9 & 0.9 \\
\hline
\end{tabular}




\begin{tabular}{|c|c|c|c|c|c|c|}
\hline $\mathrm{H}_{2} \mathrm{O}$ & $\mathrm{H}_{2} \mathrm{O}$ & & 1370.2 & 38.2 & 39.7 & -1.5 \\
\hline $\mathrm{H}_{2} \mathrm{O}$ & $\mathrm{HF}$ & & 1354.3 & 54.0 & 57.3 & -3.3 \\
\hline $\mathrm{H}_{2} \mathrm{O}$ & $\mathrm{NH}_{3}$ & & 1388.2 & 20.2 & 20.4 & -0.2 \\
\hline $\mathrm{HF}$ & $\mathrm{H}_{2} \mathrm{O}$ & & 1341.8 & 66.6 & 68.8 & -2.2 \\
\hline $\mathrm{HF}$ & $\mathrm{HF}$ & & 1325.1 & 83.2 & 86.4 & -3.2 \\
\hline $\mathrm{HF}$ & $\mathrm{NH}_{3}$ & & 1360.6 & 47.8 & 49.5 & -1.7 \\
\hline $\mathrm{NH}_{3}$ & $\mathrm{H}_{2} \mathrm{O}$ & & 1393.3 & 15.1 & 15.9 & -0.8 \\
\hline $\mathrm{NH}_{3}$ & $\mathrm{HF}$ & & 1377.6 & 30.7 & 33.5 & -2.8 \\
\hline $\mathrm{NH}_{3}$ & $\mathrm{NH}_{3}$ & & 1410.8 & -2.4 & -3.4 & 1.0 \\
\hline $\mathrm{H}_{2} \mathrm{O}$ & & $\mathrm{H}_{2} \mathrm{O}$ & 1380.9 & 27.4 & 29.5 & -2.1 \\
\hline $\mathrm{H}_{2} \mathrm{O}$ & & $\mathrm{HF}$ & 1356.4 & 51.9 & 56.4 & -4.5 \\
\hline $\mathrm{H}_{2} \mathrm{O}$ & & $\mathrm{NH}_{3}$ & 1398.3 & 10.0 & 10.1 & -0.1 \\
\hline $\mathrm{HF}$ & & $\mathrm{H}_{2} \mathrm{O}$ & 1353.2 & 55.2 & 58.6 & -3.4 \\
\hline $\mathrm{HF}$ & & $\mathrm{HF}$ & 1328.2 & 80.1 & 85.5 & -5.4 \\
\hline $\mathrm{HF}$ & & $\mathrm{NH}_{3}$ & 1371.0 & 37.4 & 39.2 & -1.8 \\
\hline $\mathrm{NH}_{3}$ & & $\mathrm{H}_{2} \mathrm{O}$ & 1403.8 & 4.6 & 5.7 & -1.1 \\
\hline $\mathrm{NH}_{3}$ & & $\mathrm{NH}_{3}$ & 1420.6 & -12.3 & -13.7 & 1.4 \\
\hline & $\mathrm{H}_{2} \mathrm{O}$ & $\mathrm{H}_{2} \mathrm{O}$ & 1378.6 & 29.8 & 30.8 & 1.0 \\
\hline
\end{tabular}




\begin{tabular}{|c|c|c|c|c|c|c|c|}
\hline & & $\mathrm{H}_{2} \mathrm{O}$ & $\mathrm{HF}$ & 1353.1 & 55.2 & 57.7 & -2.5 \\
\hline & & $\mathrm{H}_{2} \mathrm{O}$ & $\mathrm{NH}_{3}$ & 1397.5 & 10.9 & 11.4 & -0.5 \\
\hline & & $\mathrm{HF}$ & $\mathrm{H}_{2} \mathrm{O}$ & 1362.3 & 46.1 & 48.4 & -2.3 \\
\hline & & $\mathrm{HF}$ & $\mathrm{HF}$ & 1335.8 & 72.5 & 75.3 & -2.8 \\
\hline & & $\mathrm{HF}$ & $\mathrm{NH}_{3}$ & 1381.9 & 26.5 & 29.0 & -2.5 \\
\hline & & $\mathrm{NH}_{3}$ & $\mathrm{H}_{2} \mathrm{O}$ & 1397.3 & 11.0 & 11.5 & -0.5 \\
\hline & & $\mathrm{NH}_{3}$ & $\mathrm{HF}$ & 1372.5 & 35.8 & 38.4 & -2.6 \\
\hline & & $\mathrm{NH}_{3}$ & $\mathrm{NH}_{3}$ & 1414.8 & -6.4 & -7.9 & 1.5 \\
\hline $\mathrm{H}_{2} \mathrm{O}$ & $\mathrm{HF}$ & $\mathrm{H}_{2} \mathrm{O}$ & & 1342.3 & 66.1 & 75.4 & -9.3 \\
\hline $\mathrm{H}_{2} \mathrm{O}$ & $\mathrm{HF}$ & $\mathrm{HF}$ & & 1326.3 & 82.1 & 93.0 & -10.9 \\
\hline $\mathrm{HF}$ & $\mathrm{H}_{2} \mathrm{O}$ & $\mathrm{H}_{2} \mathrm{O}$ & & 1339.3 & 69.0 & 68.4 & 0.6 \\
\hline $\mathrm{HF}$ & $\mathrm{H}_{2} \mathrm{O}$ & $\mathrm{HF}$ & & 1323.8 & 84.6 & 86.0 & -1.4 \\
\hline $\mathrm{HF}$ & $\mathrm{HF}$ & $\mathrm{H}_{2} \mathrm{O}$ & & 1320.1 & 88.2 & 97.5 & -9.3 \\
\hline $\mathrm{HF}$ & $\mathrm{HF}$ & $\mathrm{HF}$ & & 1302.7 & 105.7 & 115.1 & -9.4 \\
\hline $\mathrm{HF}$ & $\mathrm{HF}$ & $\mathrm{NH}_{3}$ & & 1339.8 & 68.6 & 78.2 & -9.6 \\
\hline $\mathrm{H}_{2} \mathrm{O}$ & $\mathrm{HF}$ & & $\mathrm{H}_{2} \mathrm{O}$ & 1353.8 & 54.5 & 65.2 & -10.7 \\
\hline $\mathrm{H}_{2} \mathrm{O}$ & $\mathrm{HF}$ & & $\mathrm{HF}$ & 1332.2 & 76.2 & 92.1 & -15.9 \\
\hline $\mathrm{HF}$ & $\mathrm{H}_{2} \mathrm{O}$ & & $\mathrm{H}_{2} \mathrm{O}$ & 1353.7 & 54.6 & 58.2 & -3.6 \\
\hline
\end{tabular}




\begin{tabular}{|c|c|c|c|c|c|c|c|}
\hline $\mathrm{HF}$ & $\mathrm{H}_{2} \mathrm{O}$ & & $\mathrm{HF}$ & 1332.2 & 76.1 & 85.1 & -9.0 \\
\hline $\mathrm{HF}$ & $\mathrm{HF}$ & & $\mathrm{H}_{2} \mathrm{O}$ & 1334.4 & 73.9 & 87.3 & -13.4 \\
\hline $\mathrm{HF}$ & $\mathrm{HF}$ & & $\mathrm{HF}$ & 1311.2 & 97.1 & 114.2 & -17.1 \\
\hline $\mathrm{HF}$ & $\mathrm{HF}$ & & $\mathrm{NH}_{3}$ & 1351.3 & 57.1 & 67.9 & -10.8 \\
\hline $\mathrm{H}_{2} \mathrm{O}$ & & $\mathrm{H}_{2} \mathrm{O}$ & $\mathrm{H}_{2} \mathrm{O}$ & 1375.6 & 32.8 & 37.4 & -4.6 \\
\hline $\mathrm{H}_{2} \mathrm{O}$ & & $\mathrm{H}_{2} \mathrm{O}$ & $\mathrm{HF}$ & 1353.3 & 55.0 & 64.3 & -9.3 \\
\hline $\mathrm{H}_{2} \mathrm{O}$ & & $\mathrm{H}_{2} \mathrm{O}$ & $\mathrm{NH}_{3}$ & 1393.1 & 15.2 & 18.0 & -2.8 \\
\hline $\mathrm{H}_{2} \mathrm{O}$ & & $\mathrm{HF}$ & $\mathrm{H}_{2} \mathrm{O}$ & 1359.8 & 48.6 & 55.0 & -6.4 \\
\hline $\mathrm{H}_{2} \mathrm{O}$ & & $\mathrm{HF}$ & $\mathrm{HF}$ & 1336.8 & 71.6 & 81.9 & -10.3 \\
\hline $\mathrm{H}_{2} \mathrm{O}$ & & $\mathrm{HF}$ & $\mathrm{NH}_{3}$ & 1377.5 & 30.8 & 35.6 & -4.8 \\
\hline $\mathrm{H}_{2} \mathrm{O}$ & & $\mathrm{NH}_{3}$ & $\mathrm{H}_{2} \mathrm{O}$ & 1393.9 & 14.4 & 18.1 & -3.7 \\
\hline $\mathrm{H}_{2} \mathrm{O}$ & & $\mathrm{NH}_{3}$ & $\mathrm{HF}$ & 1372.4 & 35.9 & 45.0 & -9.1 \\
\hline $\mathrm{H}_{2} \mathrm{O}$ & & $\mathrm{NH}_{3}$ & $\mathrm{NH}_{3}$ & 1409.5 & -1.1 & -1.3 & 0.2 \\
\hline $\mathrm{HF}$ & & $\mathrm{H}_{2} \mathrm{O}$ & $\mathrm{H}_{2} \mathrm{O}$ & 1357.4 & 51.0 & 59.5 & -8.5 \\
\hline $\mathrm{HF}$ & & $\mathrm{H}_{2} \mathrm{O}$ & $\mathrm{HF}$ & 1334.7 & 73.6 & 86.4 & -12.8 \\
\hline $\mathrm{HF}$ & & $\mathrm{H}_{2} \mathrm{O}$ & $\mathrm{NH}_{3}$ & 1374.8 & 33.6 & 40.1 & -6.5 \\
\hline $\mathrm{HF}$ & & $\mathrm{HF}$ & $\mathrm{H}_{2} \mathrm{O}$ & 1340.5 & 67.9 & 77.1 & -9.2 \\
\hline $\mathrm{HF}$ & & $\mathrm{HF}$ & $\mathrm{HF}$ & 1316.5 & 91.9 & 104.0 & -12.1 \\
\hline
\end{tabular}




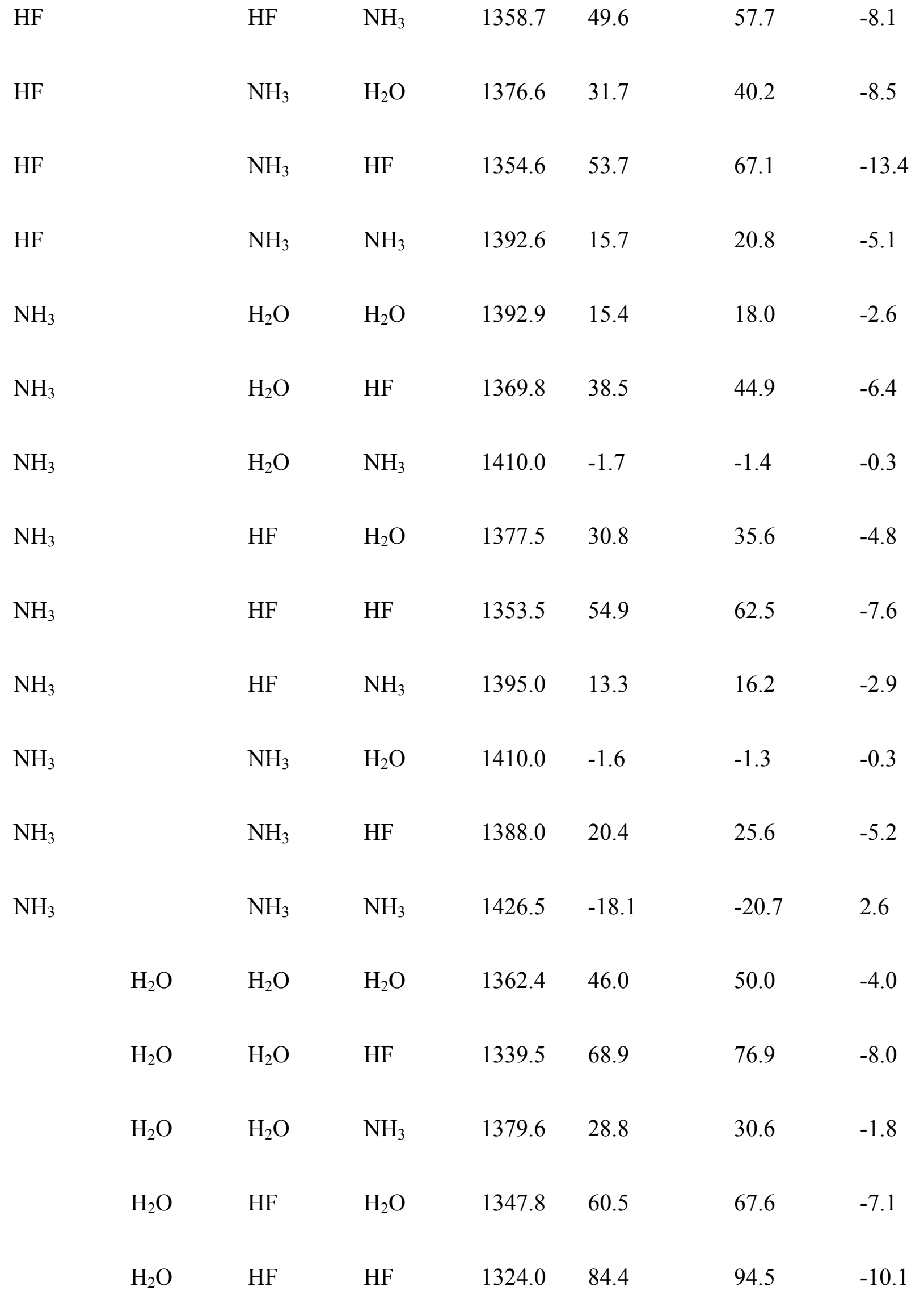




\begin{tabular}{|c|c|c|c|c|c|c|}
\hline $\mathrm{H}_{2} \mathrm{O}$ & $\mathrm{HF}$ & $\mathrm{NH}_{3}$ & 1365.7 & 42.6 & 48.2 & -5.6 \\
\hline $\mathrm{H}_{2} \mathrm{O}$ & $\mathrm{NH}_{3}$ & $\mathrm{H}_{2} \mathrm{O}$ & 1380.2 & 28.1 & 30.7 & -2.6 \\
\hline $\mathrm{H}_{2} \mathrm{O}$ & $\mathrm{NH}_{3}$ & $\mathrm{HF}$ & 1358.0 & 50.4 & 57.6 & -7.2 \\
\hline $\mathrm{H}_{2} \mathrm{O}$ & $\mathrm{NH}_{3}$ & $\mathrm{NH}_{3}$ & 1396.0 & 12.3 & 11.3 & 1.0 \\
\hline $\mathrm{HF}$ & $\mathrm{H}_{2} \mathrm{O}$ & $\mathrm{H}_{2} \mathrm{O}$ & 1335.4 & 73.0 & 79.1 & -6.1 \\
\hline $\mathrm{HF}$ & $\mathrm{H}_{2} \mathrm{O}$ & $\mathrm{HF}$ & 1311.9 & 96.5 & 106.0 & -9.5 \\
\hline $\mathrm{HF}$ & $\mathrm{H}_{2} \mathrm{O}$ & $\mathrm{NH}_{3}$ & 1353.0 & 55.4 & 59.7 & -4.3 \\
\hline $\mathrm{HF}$ & $\mathrm{HF}$ & $\mathrm{H}_{2} \mathrm{O}$ & 1320.2 & 88.2 & 96.7 & -8.5 \\
\hline $\mathrm{HF}$ & $\mathrm{HF}$ & $\mathrm{HF}$ & 1295.4 & 113.0 & 123.6 & -10.6 \\
\hline $\mathrm{HF}$ & $\mathrm{HF}$ & $\mathrm{NH}_{3}$ & 1338.3 & 70.0 & 77.3 & -7.3 \\
\hline $\mathrm{HF}$ & $\mathrm{NH}_{3}$ & $\mathrm{H}_{2} \mathrm{O}$ & 1353.8 & 54.6 & 59.8 & -5.2 \\
\hline $\mathrm{HF}$ & $\mathrm{NH}_{3}$ & $\mathrm{HF}$ & 1331.1 & 77.3 & 86.7 & -9.4 \\
\hline $\mathrm{HF}$ & $\mathrm{NH}_{3}$ & $\mathrm{NH}_{3}$ & 1369.9 & 38.4 & 40.4 & -2.0 \\
\hline $\mathrm{NH}_{3}$ & $\mathrm{H}_{2} \mathrm{O}$ & $\mathrm{H}_{2} \mathrm{O}$ & 1385.3 & 23.0 & 26.2 & -3.2 \\
\hline $\mathrm{NH}_{3}$ & $\mathrm{H}_{2} \mathrm{O}$ & $\mathrm{NH}_{3}$ & 1401.6 & 6.8 & 6.8 & 0.0 \\
\hline $\mathrm{NH}_{3}$ & $\mathrm{HF}$ & $\mathrm{H}_{2} \mathrm{O}$ & 1370.6 & 37.7 & 43.8 & -6.1 \\
\hline $\mathrm{NH}_{3}$ & $\mathrm{HF}$ & $\mathrm{NH}_{3}$ & 1387.4 & 20.9 & 24.4 & -3.5 \\
\hline $\mathrm{NH}_{3}$ & $\mathrm{NH}_{3}$ & $\mathrm{H}_{2} \mathrm{O}$ & 1401.7 & 6.7 & 6.9 & -0.2 \\
\hline
\end{tabular}




\begin{tabular}{|c|c|c|c|c|c|c|c|}
\hline & $\mathrm{NH}_{3}$ & $\mathrm{NH}_{3}$ & $\mathrm{HF}$ & 1379.6 & 28.7 & 33.8 & -5.1 \\
\hline & $\mathrm{NH}_{3}$ & $\mathrm{NH}_{3}$ & $\mathrm{NH}_{3}$ & 1417.3 & -9.0 & -12.5 & 3.5 \\
\hline $\mathrm{H}_{2} \mathrm{O}$ & $\mathrm{HF}$ & $\mathrm{H}_{2} \mathrm{O}$ & $\mathrm{H}_{2} \mathrm{O}$ & 1337.2 & 71.2 & 85.7 & -14.5 \\
\hline $\mathrm{H}_{2} \mathrm{O}$ & $\mathrm{HF}$ & $\mathrm{H}_{2} \mathrm{O}$ & $\mathrm{HF}$ & 1316.5 & 91.9 & 112.6 & -20.7 \\
\hline $\mathrm{H}_{2} \mathrm{O}$ & $\mathrm{HF}$ & $\mathrm{HF}$ & $\mathrm{H}_{2} \mathrm{O}$ & 1322.3 & 86.1 & 103.3 & -17.2 \\
\hline $\mathrm{H}_{2} \mathrm{O}$ & $\mathrm{HF}$ & $\mathrm{HF}$ & $\mathrm{HF}$ & 1300.6 & 107.8 & 130.2 & -22.4 \\
\hline $\mathrm{HF}$ & $\mathrm{H}_{2} \mathrm{O}$ & $\mathrm{H}_{2} \mathrm{O}$ & $\mathrm{HF}$ & 1316.9 & 91.4 & 105.6 & -14.2 \\
\hline $\mathrm{HF}$ & $\mathrm{H}_{2} \mathrm{O}$ & $\mathrm{HF}$ & $\mathrm{H}_{2} \mathrm{O}$ & 1322.2 & 86.2 & 96.3 & -10.1 \\
\hline $\mathrm{HF}$ & $\mathrm{H}_{2} \mathrm{O}$ & $\mathrm{HF}$ & $\mathrm{HF}$ & 1301.1 & 107.2 & 123.2 & -16.0 \\
\hline $\mathrm{HF}$ & $\mathrm{HF}$ & $\mathrm{H}_{2} \mathrm{O}$ & $\mathrm{H}_{2} \mathrm{O}$ & 1318.0 & 90.4 & 107.8 & -17.4 \\
\hline $\mathrm{HF}$ & $\mathrm{HF}$ & $\mathrm{H}_{2} \mathrm{O}$ & $\mathrm{HF}$ & 1296.2 & 112.1 & 134.7 & -22.6 \\
\hline $\mathrm{HF}$ & $\mathrm{HF}$ & $\mathrm{HF}$ & $\mathrm{H}_{2} \mathrm{O}$ & 1301.6 & 106.7 & 125.4 & -18.7 \\
\hline $\mathrm{HF}$ & $\mathrm{HF}$ & $\mathrm{HF}$ & $\mathrm{HF}$ & 1278.2 & 130.2 & 152.3 & -22.1 \\
\hline $\mathrm{HF}$ & $\mathrm{HF}$ & $\mathrm{HF}$ & $\mathrm{NH}_{3}$ & 1318.8 & 89.6 & 106.0 & -16.4 \\
\hline $\mathrm{HF}$ & $\mathrm{HF}$ & $\mathrm{NH}_{3}$ & $\mathrm{HF}$ & 1315.8 & 92.6 & 115.4 & -22.8 \\
\hline $\mathrm{HF}$ & $\mathrm{HF}$ & $\mathrm{NH}_{3}$ & $\mathrm{NH}_{3}$ & 1352.1 & 56.2 & 69.1 & -12.9 \\
\hline
\end{tabular}

${ }^{\mathrm{a} B} 3 \mathrm{LYP} / 6-311+\mathrm{G}(2 \mathrm{~d}, \mathrm{p})$ single-point calculations on $\mathrm{B} 3 \mathrm{LYP} / 6-31+\mathrm{G}(\mathrm{d}, \mathrm{p})$ geometries. See Figure 1 for structure, atomic numbering and notation for 8-oxoguanine and its complexes. ${ }^{\mathrm{b}}$ The calculated (N9) acidities of isolated 8-oxoguanine $\left(1408.4 \mathrm{~kJ} \mathrm{~mol}^{-1}\right)$ minus the calculated acidity of the 8-oxoguanine 
complex. A positive value represents an increase in the acidity. ${ }^{\mathrm{c}}$ The sum of the effects of the individual hydrogen bonds on the acidity. ${ }^{\mathrm{d}}$ The difference between the additive and the calculated effect of more than one molecule, where a positive value indicates that the effects are greater than additive.

Table S5: Cartesian coordinates for the neutral complexes between ammonia, water or hydrogen fluoride at each binding site within the four nucleobases considered in the present work (see Figure 1).

\begin{tabular}{llll}
\multicolumn{4}{c}{ Adenine with hydrogen fluoride at $\mathrm{N}$ (N6) } \\
6 & 0.19638 & 0.05753 & -1.27246 \\
7 & -0.92799 & 0.06338 & -2.01773 \\
6 & -2.11991 & 0.01279 & -1.40357 \\
6 & 0.05040 & -0.00387 & 0.13634 \\
7 & 1.39273 & 0.10918 & -1.88834 \\
1 & 1.40800 & 0.14989 & -2.89583 \\
9 & 3.33916 & 0.05358 & 0.33561 \\
6 & -1.25552 & -0.05156 & 0.62700 \\
7 & 0.95749 & -0.02930 & 1.18664 \\
1 & -2.98436 & 0.02065 & -2.06227 \\
7 & -1.11628 & -0.10560 & 2.00161 \\
7 & -2.38309 & -0.04604 & -0.09182 \\
6 & 0.22614 & -0.08946 & 2.27797 \\
1 & 0.61426 & -0.12390 & 3.28586 \\
1 & -1.87326 & -0.14948 & 2.66901 \\
1 & 2.25956 & 0.10873 & -1.35922 \\
1 & 2.47835 & 0.01792 & 0.81779
\end{tabular}

Adenine with hydrogen fluoride at N1(N6)

$\begin{array}{llll}6 & 0.19526 & -0.77158 & -0.46471 \\ 7 & 0.00855 & 0.07877 & -1.50268 \\ 6 & -0.30808 & 1.36903 & -1.26774 \\ 6 & 0.04470 & -0.24773 & 0.83757 \\ 7 & 0.51003 & -2.05521 & -0.71776 \\ 1 & 0.60435 & -2.36654 & -1.67773 \\ 9 & 0.38700 & -1.32888 & -3.57250 \\ 6 & -0.28525 & 1.10872 & 0.92766 \\ 7 & 0.16100 & -0.82274 & 2.09008 \\ 1 & -0.44051 & 1.98335 & -2.15354 \\ 7 & -0.36973 & 1.35481 & 2.27826 \\ 7 & -0.47353 & 1.96359 & -0.09062 \\ 6 & -0.09276 & 0.16259 & 2.91867 \\ 1 & -0.09380 & 0.08516 & 3.99724 \\ 1 & 0.20980 & -0.64979 & -2.87529 \\ 1 & 0.65209 & -2.69571 & 0.04708 \\ 1 & -0.59248 & 2.24280 & 2.70508\end{array}$


Adenine with water at N7(N6)

$\begin{array}{llll}\mathrm{N} & -0.94830 & 0.12240 & 1.99254 \\ \mathrm{C} & 0.16554 & 0.18065 & 1.23059 \\ \mathrm{C} & 0.02071 & -0.01334 & -0.16808 \\ \mathrm{C} & -1.27941 & -0.25319 & -0.62496 \\ \mathrm{~N} & -2.39581 & -0.31458 & 0.11038 \\ \mathrm{C} & -2.13116 & -0.11414 & 1.40705 \\ \mathrm{H} & -2.98562 & -0.14784 & 2.07833 \\ \mathrm{~N} & 1.34671 & 0.42058 & 1.83155 \\ \mathrm{H} & 2.22105 & 0.45147 & 1.30765 \\ \mathrm{~N} & 0.91873 & -0.02790 & -1.22909 \\ \mathrm{~N} & -1.15139 & -0.41337 & -1.99012 \\ \mathrm{H} & -1.90575 & -0.60481 & -2.63355 \\ \mathrm{H} & 0.55511 & -0.35235 & -3.30127 \\ \mathrm{C} & 0.18218 & -0.26886 & -2.28995 \\ \mathrm{O} & 3.48890 & 0.38357 & -0.16753 \\ \mathrm{H} & 2.72806 & 0.24719 & -0.77763 \\ \mathrm{H} & 1.34782 & 0.51494 & 2.83567 \\ \mathrm{H} & 3.99097 & 1.13448 & -0.50445\end{array}$

Adenine with water at N1(N6)

$\begin{array}{llll}\mathrm{N} & 0.16385 & -0.09421 & 1.48967 \\ \mathrm{C} & -0.70303 & -0.22527 & 0.45733 \\ \mathrm{C} & -0.21698 & 0.02804 & -0.84600 \\ \mathrm{C} & 1.12907 & 0.39148 & -0.94822 \\ \mathrm{~N} & 2.00530 & 0.52625 & 0.05907 \\ \mathrm{C} & 1.43698 & 0.26442 & 1.23638 \\ \mathrm{H} & 2.07886 & 0.34841 & 2.10949 \\ \mathrm{~N} & -1.97806 & -0.58339 & 0.70421 \\ \mathrm{H} & -2.61558 & -0.70300 & -0.06690 \\ \mathrm{~N} & -0.82305 & -0.01482 & -2.09013 \\ \mathrm{~N} & 1.33781 & 0.57337 & -2.29709 \\ \mathrm{H} & 2.20974 & 0.84198 & -2.72992 \\ \mathrm{H} & 0.02807 & 0.38798 & -3.99814 \\ \mathrm{C} & 0.13445 & 0.31533 & -2.92442 \\ \mathrm{O} & -1.57278 & -0.84966 & 3.58325 \\ \mathrm{H} & -1.70425 & -0.26080 & 4.33536 \\ \mathrm{H} & -2.27153 & -0.76750 & 1.66023 \\ \mathrm{H} & -0.76836 & -0.53873 & 3.10848\end{array}$

Adenine with ammonia at N7(N6)

$\begin{array}{llll}6 & 0.13882 & -0.47162 & -1.11903 \\ 7 & 0.00965 & 0.36779 & -2.17179 \\ 6 & -0.26058 & 1.66183 & -1.94930 \\ 6 & -0.02129 & 0.07102 & 0.18362 \\ 7 & 0.41062 & -1.76746 & -1.36166 \\ 1 & 0.50268 & -2.04251 & -2.32798 \\ 1 & 0.47316 & -2.64767 & 1.57234 \\ 6 & -0.30062 & 1.44121 & 0.24688 \\ 7 & 0.04068 & -0.47652 & 1.46016\end{array}$




$\begin{array}{llll}1 & -0.35114 & 2.27933 & -2.83970 \\ 7 & -0.40813 & 1.71823 & 1.59394 \\ 7 & -0.43268 & 2.29022 & -0.78025 \\ 6 & -0.19441 & 0.53451 & 2.26428 \\ 1 & -0.22435 & 0.47806 & 3.34384 \\ 7 & 0.66464 & -3.50217 & 1.04402 \\ 1 & 0.52024 & -2.46109 & -0.61246 \\ 1 & 1.56445 & -3.86994 & 1.34059 \\ 1 & -0.04379 & -4.19195 & 1.27898 \\ 1 & -0.60619 & 2.62350 & 1.99464\end{array}$

Adenine with ammonia at N7(C8)

$\begin{array}{llll}6 & 0.20590 & -0.70203 & -1.49523 \\ 7 & 0.08652 & 0.08473 & -2.57989 \\ 6 & -0.19928 & 1.38655 & -2.40360 \\ 6 & 0.03156 & -0.12840 & -0.21786 \\ 7 & 0.47430 & -2.02003 & -1.67247 \\ 1 & 0.67536 & -2.35128 & -2.60300 \\ 1 & 0.31331 & -2.03031 & 3.04290 \\ 6 & -0.26075 & 1.24026 & -0.19748 \\ 7 & 0.09226 & -0.64580 & 1.06729 \\ 1 & -0.28480 & 1.97289 & -3.31477 \\ 7 & -0.37919 & 1.54942 & 1.14057 \\ 7 & -0.38942 & 2.04848 & -1.25748 \\ 6 & -0.15801 & 0.38506 & 1.84516 \\ 1 & -0.18934 & 0.33568 & 2.92628 \\ 7 & 0.25818 & -1.89738 & 4.05203 \\ 1 & 0.66443 & -2.60870 & -0.87766 \\ 1 & -0.45310 & -2.52663 & 4.41390 \\ 1 & 1.14828 & -2.17693 & 4.45504 \\ 1 & -0.58935 & 2.46073 & 1.52109\end{array}$

Adenine with ammonia at N1(C2)

$\begin{array}{llll}6 & -0.92790 & -0.24184 & -0.44392 \\ 7 & 0.02564 & -0.03476 & -1.37088 \\ 6 & 1.27120 & 0.28378 & -0.96446 \\ 6 & -0.58255 & -0.11479 & 0.91842 \\ 7 & -2.17983 & -0.56797 & -0.84524 \\ 1 & -2.38644 & -0.62106 & -1.82978 \\ 1 & 0.38983 & -0.01870 & -3.60796 \\ 6 & 0.74933 & 0.22084 & 1.18467 \\ 7 & -1.31113 & -0.25651 & 2.08834 \\ 1 & 1.99165 & 0.43694 & -1.76341 \\ 7 & 0.82199 & 0.28166 & 2.55936 \\ 7 & 1.71931 & 0.43170 & 0.28545 \\ 6 & -0.43948 & -0.01254 & 3.03850 \\ 1 & -0.65242 & -0.03134 & 4.09865 \\ 7 & 0.93705 & 0.08804 & -4.46213 \\ 1 & -2.91199 & -0.69963 & -0.16594 \\ 1 & 0.90138 & -0.78522 & -4.97980\end{array}$




$\begin{array}{llll}1 & 0.50648 & 0.80884 & -5.03388 \\ 1 & 1.64678 & 0.50235 & 3.09852\end{array}$

Adenine with ammonia at N1(N6)

$\begin{array}{llll}6 & -0.62868 & -0.16188 & -0.48603 \\ 7 & 0.27849 & 0.05259 & -1.46851 \\ 6 & 1.54052 & 0.36933 & -1.12847 \\ 6 & -0.19288 & -0.03945 & 0.85532 \\ 7 & -1.89617 & -0.47985 & -0.81261 \\ 1 & -2.16226 & -0.55484 & -1.79895 \\ 1 & -0.87699 & -0.24771 & -3.47731 \\ 6 & 1.15058 & 0.29607 & 1.04498 \\ 7 & -0.84973 & -0.18941 & 2.06609 \\ 1 & 2.22015 & 0.52904 & -1.96217 \\ 7 & 1.30592 & 0.34933 & 2.41328 \\ 7 & 2.06771 & 0.51346 & 0.09062 \\ 6 & 0.07544 & 0.04988 & 2.96525 \\ 1 & -0.07396 & 0.02429 & 4.03608 \\ 7 & -1.82392 & -0.48859 & -3.77736 \\ 1 & -2.56552 & -0.63761 & -0.07586 \\ 1 & -2.17398 & 0.26275 & -4.36569 \\ 1 & -1.77473 & -1.32923 & -4.34663 \\ 1 & 2.16129 & 0.56690 & 2.90354\end{array}$

8oxoadenine with hydrogen fluoride at O8(N7)

$\begin{array}{llll}6 & 0.07843 & -0.03875 & 1.78346 \\ 8 & 0.34017 & -0.33913 & 2.95737 \\ 7 & -0.70312 & 1.01697 & 1.35046 \\ 7 & 0.50783 & -0.68792 & 0.64485 \\ 1 & 1.13695 & -1.47968 & 0.72123 \\ 6 & 0.00947 & -0.03708 & -0.48645 \\ 6 & -0.75602 & 1.04098 & -0.03469 \\ 1 & -1.74144 & 2.33075 & -2.79742 \\ 6 & 0.09129 & -0.22116 & -1.87063 \\ 7 & -1.40061 & 1.91326 & -0.80202 \\ 7 & -0.55294 & 0.65574 & -2.66832 \\ 7 & 0.82312 & -1.20967 & -2.47266 \\ 6 & -1.24372 & 1.65282 & -2.11008 \\ 1 & 0.67091 & -1.31887 & -3.46578 \\ 1 & 1.05270 & -2.04871 & -1.96213 \\ 9 & 1.80440 & -2.33733 & 2.54528 \\ 1 & 1.27336 & -1.60543 & 2.90557 \\ 1 & -1.15009 & 1.67138 & 1.97622\end{array}$

8oxoadenine with hydrogen fluoride at N1(N6)

$\begin{array}{llll}6 & 2.41753 & 1.18312 & -0.02586 \\ 8 & 3.56040 & 1.57778 & 0.10826 \\ 7 & 1.45359 & 1.63472 & -0.93846 \\ 7 & 1.76906 & 0.17712 & 0.69608 \\ 1 & 2.27607 & -0.39061 & 1.35713\end{array}$




$\begin{array}{llll}6 & 0.47235 & -0.00581 & 0.21472 \\ 6 & 0.28174 & 0.92271 & -0.81386 \\ 1 & -2.73530 & 0.28019 & -1.71034 \\ 6 & -0.60050 & -0.83930 & 0.53607 \\ 7 & -0.83263 & 1.07150 & -1.53065 \\ 7 & -1.73744 & -0.69709 & -0.19608 \\ 7 & -0.57740 & -1.80861 & 1.49442 \\ 6 & -1.79672 & 0.22251 & -1.16766 \\ 1 & -1.45866 & -2.26886 & 1.70274 \\ 1 & 0.10353 & -1.76573 & 2.23575 \\ 9 & -3.39892 & -2.30455 & 0.85453 \\ 1 & -2.88553 & -1.64813 & 0.32762 \\ 1 & 1.63420 & 2.36911 & -1.60739\end{array}$

\begin{tabular}{llll}
\multicolumn{5}{l}{ 8oxoadenine with water at O8(N7) } \\
6 & 0.04452 & 0.02650 & 1.81794 \\
8 & -0.28662 & 0.10111 & 3.00202 \\
7 & 1.32126 & -0.21078 & 1.31495 \\
7 & -0.77244 & 0.15963 & 0.70775 \\
1 & -1.77500 & 0.28323 & 0.83570 \\
6 & -0.02239 & -0.00365 & -0.45616 \\
6 & 1.30081 & -0.23718 & -0.06812 \\
1 & 2.74883 & -0.57516 & -2.90897 \\
6 & -0.28222 & 0.03266 & -1.82823 \\
7 & 2.32500 & -0.44522 & -0.89010 \\
7 & 0.74152 & -0.18078 & -2.68103 \\
7 & -1.53151 & 0.22504 & -2.36568 \\
6 & 1.95937 & -0.40719 & -2.18192 \\
1 & -1.54319 & 0.39769 & -3.36174 \\
8 & -2.96908 & 0.59789 & 2.40422 \\
1 & -2.17141 & 0.45365 & 2.95584 \\
1 & -3.70749 & 0.17049 & 2.85290 \\
1 & -2.22400 & 0.72501 & -1.82779
\end{tabular}

\begin{tabular}{llll}
\multicolumn{4}{l}{ 8oxoadenine with water at N1(N6) } \\
6 & -0.98131 & -0.93659 & 2.34620 \\
8 & -1.36822 & -1.14870 & 3.48167 \\
7 & -1.04008 & -1.79929 & 1.24438 \\
7 & -0.37895 & 0.22209 & 1.85085 \\
1 & -0.31917 & 1.05336 & 2.41788 \\
6 & -0.12058 & 0.08893 & 0.48429 \\
6 & -0.53596 & -1.19250 & 0.11267 \\
1 & 0.17654 & -1.20983 & -3.01479 \\
6 & 0.45098 & 0.89477 & -0.50443 \\
7 & -0.45392 & -1.70841 & -1.11281 \\
7 & 0.53099 & 0.38462 & -1.76081 \\
7 & 0.88792 & 2.17227 & -0.29632 \\
6 & 0.08471 & -0.85449 & -1.99226 \\
1 & 1.40432 & 2.60714 & -1.06045 \\
8 & 2.05864 & 2.44872 & -2.95608
\end{tabular}




$\begin{array}{llll}1 & 1.53041 & 1.62403 & -2.87636 \\ 1 & 1.77211 & 2.89552 & -3.76119 \\ 1 & 1.13621 & 2.45759 & 0.63820 \\ 1 & -1.43264 & -2.72770 & 1.29620\end{array}$

\begin{tabular}{llll}
\multicolumn{4}{l}{ 8oxoadenine with ammonia at O8(N7) } \\
6 & -1.84448 & -0.19637 & 0.17163 \\
8 & -3.00845 & -0.01181 & 0.51521 \\
7 & -1.36860 & -1.16728 & -0.71647 \\
7 & -0.72312 & 0.51118 & 0.57752 \\
7 & -2.01142 & 2.44833 & 2.26695 \\
6 & 0.41065 & 0.00405 & -0.05281 \\
6 & 0.00008 & -1.05680 & -0.86843 \\
1 & 2.78760 & -1.98744 & -2.15503 \\
6 & 1.77569 & 0.29595 & -0.03756 \\
7 & 0.79605 & -1.79795 & -1.63474 \\
7 & 2.60271 & -0.44182 & -0.80815 \\
7 & 2.32751 & 1.32945 & 0.68312 \\
6 & 2.08309 & -1.42287 & -1.55067 \\
1 & 3.33599 & 1.30887 & 0.74973 \\
1 & 1.84154 & 1.66318 & 1.50240 \\
1 & -1.96774 & -1.83363 & -1.18028 \\
1 & -2.19097 & 3.40589 & 1.97535 \\
1 & -2.01961 & 2.43368 & 3.28369 \\
1 & -0.84050 & 1.31443 & 1.21051 \\
1 & -2.79092 & 1.87238 & 1.94140
\end{tabular}

\begin{tabular}{llll}
\multicolumn{4}{l}{ 8oxoadenine with ammonia at N1(N6) } \\
6 & -2.68100 & -0.47068 & 0.24132 \\
8 & -3.87819 & -0.45806 & 0.47376 \\
7 & -1.95683 & -1.43044 & -0.47473 \\
7 & -1.72593 & 0.46967 & 0.63058 \\
7 & 4.07896 & 1.97758 & 0.65433 \\
6 & -0.46735 & 0.12148 & 0.12880 \\
6 & -0.62273 & -1.08225 & -0.56137 \\
1 & 2.37164 & -1.62364 & -1.56998 \\
6 & 0.81963 & 0.66788 & 0.19643 \\
7 & 0.34847 & -1.75027 & -1.18166 \\
7 & 1.81925 & 0.00450 & -0.43953 \\
7 & 1.12206 & 1.84111 & 0.82216 \\
6 & 1.53544 & -1.13399 & -1.07771 \\
1 & 2.11733 & 2.07446 & 0.92524 \\
1 & 0.48450 & 2.21018 & 1.51006 \\
1 & 4.73493 & 1.69302 & 1.37678 \\
1 & 4.54736 & 2.65950 & 0.06369 \\
1 & 3.85569 & 1.16035 & 0.08416 \\
1 & -2.00487 & 1.33450 & 1.06653 \\
1 & -2.38690 & -2.24370 & -0.88943
\end{tabular}

guanine with hydrogen fluoride at O6-N7 


$\begin{array}{llll}7 & 0.22282 & -0.66754 & 2.08480 \\ 1 & 3.55740 & 0.12378 & -2.29148 \\ 1 & 4.14210 & -0.18517 & -0.71701 \\ 7 & 1.10666 & 0.43162 & -1.60943 \\ 7 & -1.57926 & -0.15808 & 0.87500 \\ 8 & -1.11407 & 0.75730 & -2.09899 \\ 6 & -0.44518 & -0.00019 & 0.10080 \\ 7 & 1.99105 & -0.29987 & 0.45938 \\ 6 & 0.69115 & -0.31419 & 0.84339 \\ 6 & -0.28793 & 0.42461 & -1.26642 \\ 6 & 2.15043 & 0.09003 & -0.78603 \\ 6 & -1.14908 & -0.55582 & 2.04918 \\ 9 & -4.19153 & 0.04737 & 0.74077 \\ 1 & -1.77573 & -0.77614 & 2.90137 \\ 7 & 3.41685 & 0.21487 & -1.29589 \\ 1 & 1.28855 & 0.77833 & -2.54458 \\ 1 & -3.23090 & 0.01325 & 0.66485 \\ 1 & 0.79176 & -0.95242 & 2.86929\end{array}$

\begin{tabular}{llll}
\multicolumn{4}{c}{ guanine with hydrogen fluoride at $\mathrm{N} 3(\mathrm{~N} 2)$} \\
7 & -0.22708 & 1.25070 & 1.82084 \\
1 & 2.06869 & -1.60530 & -2.39788 \\
1 & 2.97172 & -0.54786 & -1.37313 \\
7 & -0.23687 & -1.00419 & -1.43426 \\
7 & -2.27882 & 0.53694 & 1.25811 \\
8 & -2.51197 & -1.26702 & -1.30485 \\
6 & -1.34744 & 0.05780 & 0.35738 \\
7 & 1.10581 & 0.22789 & 0.05666 \\
6 & -0.06749 & 0.49376 & 0.69356 \\
6 & -1.51588 & -0.77256 & -0.80721 \\
6 & 0.97630 & -0.53033 & -1.02164 \\
6 & -1.58364 & 1.24026 & 2.11364 \\
1 & -0.30271 & -1.54732 & -2.28762 \\
1 & 2.61527 & 0.56140 & 0.38095 \\
7 & 2.07657 & -0.81871 & -1.76756 \\
9 & 3.59667 & 0.61129 & 0.36291 \\
1 & -1.98198 & 1.76998 & 2.96749 \\
1 & 0.50639 & 1.72172 & 2.33182
\end{tabular}

\begin{tabular}{llll}
\multicolumn{4}{c}{ guanine with hydrogen fluoride at } \\
7 & 1.38684 & 0.51488 & 2.32879 \\
1 & -0.70028 & -3.24630 & -1.20773 \\
1 & 0.32290 & -3.67073 & 0.10297 \\
7 & -0.48675 & -0.72472 & -0.92191 \\
7 & 0.47227 & 2.19146 & 1.14621 \\
8 & -1.06199 & 1.43126 & -1.46426 \\
6 & 0.22107 & 0.97994 & 0.52882 \\
7 & 0.74970 & -1.40267 & 0.98162 \\
6 & 0.78435 & -0.07786 & 1.25249 \\
6 & -0.48607 & 0.66610 & -0.66882
\end{tabular}




$\begin{array}{llll}6 & 0.10074 & -1.67961 & -0.12948 \\ 6 & 1.16605 & 1.87980 & 2.20932 \\ 1 & -0.96012 & -0.98704 & -1.78888 \\ 1 & -1.68450 & 0.66841 & -2.60392 \\ 7 & 0.02851 & -2.97349 & -0.56521 \\ 9 & -1.96561 & -0.07531 & -3.19206 \\ 1 & 1.54445 & 2.58039 & 2.94065 \\ 1 & 1.89316 & 0.03456 & 3.05892\end{array}$

\begin{tabular}{llll}
\multicolumn{4}{l}{ guanine with water at O6-N7 } \\
7 & 0.77991 & 2.02607 & -1.01515 \\
1 & 2.84558 & -2.10723 & 2.07051 \\
1 & 3.82648 & -0.93861 & 1.30738 \\
7 & 0.66516 & -1.42407 & 0.90992 \\
7 & -1.28303 & 1.16490 & -0.88302 \\
8 & -1.60038 & -1.58481 & 0.60001 \\
6 & -0.38122 & 0.32819 & -0.25190 \\
7 & 2.06328 & 0.33868 & 0.17612 \\
6 & 0.91096 & 0.84914 & -0.32509 \\
6 & -0.58219 & -0.92820 & 0.41621 \\
6 & 1.88919 & -0.81241 & 0.78467 \\
6 & -0.56143 & 2.16393 & -1.32535 \\
1 & 0.59541 & -2.33927 & 1.34045 \\
8 & -4.03145 & -0.15026 & -0.57238 \\
7 & 2.97564 & -1.48361 & 1.28699 \\
1 & -3.33202 & 0.47617 & -0.81939 \\
1 & -0.93660 & 3.01622 & -1.87398 \\
1 & -3.53386 & -0.84475 & -0.11444 \\
1 & 1.53106 & 2.66030 & -1.24687
\end{tabular}

\begin{tabular}{llll}
\multicolumn{5}{l}{ guanine with water at $\mathrm{N} 3(\mathrm{~N} 2)$} \\
7 & 0.27338 & 1.73429 & -1.41577 \\
1 & 1.24225 & -2.43227 & 2.15896 \\
1 & 2.51341 & -1.66289 & 1.27242 \\
7 & -0.68824 & -1.25203 & 0.98755 \\
7 & -1.94150 & 1.52178 & -1.12983 \\
8 & -2.92407 & -0.80473 & 0.74100 \\
6 & -1.27428 & 0.55315 & -0.40400 \\
7 & 1.09820 & -0.08231 & -0.02262 \\
6 & 0.10620 & 0.66928 & -0.57003 \\
6 & -1.78185 & -0.49312 & 0.44423 \\
6 & 0.64928 & -1.04981 & 0.76079 \\
6 & -0.99575 & 2.20700 & -1.71762 \\
1 & -0.98898 & -2.03184 & 1.56088 \\
8 & 3.87130 & -0.41121 & 0.49064 \\
7 & 1.52783 & -1.91448 & 1.34213 \\
1 & 4.48379 & -0.61162 & -0.22690 \\
1 & -1.14369 & 3.05376 & -2.37318 \\
1 & 3.05038 & -0.06094 & 0.08315 \\
1 & 1.15571 & 2.10362 & -1.73898
\end{tabular}




\begin{tabular}{llll}
\multicolumn{5}{l}{ guanine with water at O6(N1) } \\
7 & 1.03440 & 1.90083 & -1.75911 \\
1 & 1.94351 & -2.17702 & 1.90535 \\
1 & 3.18504 & -1.51588 & 0.93168 \\
7 & 0.02173 & -0.95296 & 0.78298 \\
7 & -1.17109 & 1.83561 & -1.34482 \\
8 & -2.19009 & -0.34305 & 0.65682 \\
6 & -0.52273 & 0.84883 & -0.62475 \\
7 & 1.82461 & 0.08443 & -0.35214 \\
6 & 0.85334 & 0.87409 & -0.87030 \\
6 & -1.03054 & -0.13565 & 0.28508 \\
6 & 1.34984 & -0.82468 & 0.47287 \\
6 & -0.21887 & 2.44037 & -2.00692 \\
1 & -0.29599 & -1.70633 & 1.40111 \\
8 & -1.78643 & -2.43385 & 2.38417 \\
7 & 2.20525 & -1.73877 & 1.03401 \\
1 & -2.25885 & -1.73703 & 1.87368 \\
1 & -0.35380 & 3.27307 & -2.68318 \\
1 & -2.31733 & -3.23691 & 2.32923 \\
1 & 1.91912 & 2.19342 & -2.14813
\end{tabular}

\begin{tabular}{llll}
\multicolumn{4}{l}{ guanine with ammonia at O6-N7 } \\
7 & 1.06592 & -0.04035 & 2.16221 \\
1 & -1.35616 & -3.55679 & -1.38056 \\
1 & -0.61168 & -4.06397 & 0.06396 \\
7 & -0.48031 & -1.14933 & -1.29944 \\
7 & 0.81691 & 1.66690 & 0.72988 \\
8 & -0.39458 & 0.99958 & -2.09394 \\
6 & 0.37748 & 0.49231 & 0.14773 \\
7 & 0.20067 & -1.88746 & 0.84129 \\
6 & 0.52458 & -0.58243 & 1.02625 \\
6 & -0.17439 & 0.24368 & -1.15697 \\
6 & -0.29826 & -2.12384 & -0.35000 \\
6 & 1.21825 & 1.31525 & 1.92532 \\
1 & -0.82170 & -1.39528 & -2.22163 \\
7 & -1.47525 & 3.70632 & -0.64787 \\
7 & -0.61546 & -3.41245 & -0.70896 \\
1 & -1.32860 & 3.09559 & -1.44938 \\
1 & 1.62709 & 1.98057 & 2.67282 \\
1 & -0.66234 & 3.56920 & -0.05075 \\
1 & -1.46121 & 4.66647 & -0.98077 \\
1 & 1.29794 & -0.54762 & 3.00405
\end{tabular}

\begin{tabular}{llll}
\multicolumn{4}{l}{ guanine with ammonia at N3(N2) } \\
7 & 1.12718 & 0.96384 & 1.74934 \\
1 & -0.96842 & -2.63593 & -1.95483 \\
1 & 0.23326 & -3.11846 & -0.78817 \\
7 & -0.98849 & -0.16338 & -1.38690 \\
7 & -0.07483 & 2.63691 & 0.86141
\end{tabular}




$\begin{array}{llll}8 & -1.83084 & 1.95253 & -1.65604 \\ 6 & -0.28446 & 1.47073 & 0.14831 \\ 7 & 0.52431 & -0.87954 & 0.28563 \\ 6 & 0.45668 & 0.41584 & 0.68555 \\ 6 & -1.10586 & 1.21474 & -1.00465 \\ 6 & -0.22220 & -1.12968 & -0.77738 \\ 6 & 0.76738 & 2.30262 & 1.80409 \\ 1 & -1.51567 & -0.39180 & -2.22163 \\ 7 & 1.31037 & -3.98283 & 0.60256 \\ 7 & -0.22188 & -2.37289 & -1.33039 \\ 1 & 0.91062 & -4.58170 & 1.31988 \\ 1 & 1.16303 & 2.96566 & 2.56087 \\ 1 & 1.42389 & -3.04651 & 0.99170 \\ 1 & 2.23086 & -4.34676 & 0.37153 \\ 1 & 1.76331 & 0.47491 & 2.36181\end{array}$

\begin{tabular}{llll}
\multicolumn{4}{l}{ guanine with ammonia at O6(N1) } \\
7 & 1.47457 & 1.02079 & 2.17300 \\
1 & -0.16443 & -3.30865 & -0.90788 \\
1 & 1.02614 & -3.44339 & 0.31667 \\
7 & -0.41925 & -0.79392 & -0.78914 \\
7 & 0.14254 & 2.40079 & 1.00738 \\
8 & -1.45782 & 1.16417 & -1.38836 \\
6 & 0.07531 & 1.12207 & 0.48401 \\
7 & 1.10548 & -1.07804 & 1.00479 \\
6 & 0.89937 & 0.24798 & 1.19755 \\
6 & -0.67742 & 0.59811 & -0.62318 \\
6 & 0.41865 & -1.54867 & -0.01583 \\
6 & 0.98110 & 2.30583 & 2.00712 \\
1 & -0.92275 & -1.21943 & -1.58557 \\
7 & -2.13059 & -1.27862 & -3.09698 \\
7 & 0.56952 & -2.86607 & -0.37454 \\
1 & -1.77537 & -1.35434 & -4.04687 \\
1 & 1.28165 & 3.11687 & 2.65589 \\
1 & -2.23951 & -0.28401 & -2.88019 \\
1 & -3.05372 & -1.70485 & -3.08209 \\
1 & 2.13253 & 0.69809 & 2.86748
\end{tabular}

8oxoguanine with hydrogen fluoride at O6(N7)

$\begin{array}{llll}6 & -0.43792 & -0.55726 & 2.34353 \\ 8 & -0.49429 & -0.78232 & 3.53860 \\ 7 & -1.48090 & -0.75677 & 1.40233 \\ 7 & 0.61225 & -0.06427 & 1.58953 \\ 1 & 2.89338 & 0.96336 & 0.27481 \\ 6 & 0.23847 & 0.03632 & 0.25168 \\ 6 & -1.07844 & -0.40052 & 0.14496 \\ 1 & -1.33550 & -0.10583 & -4.11926 \\ 6 & 0.92007 & 0.47719 & -0.89843 \\ 7 & -1.81848 & -0.47509 & -0.98109 \\ 8 & 2.08631 & 0.91226 & -1.02792\end{array}$




$\begin{array}{llll}7 & 0.10112 & 0.39403 & -2.04745 \\ 6 & -1.19185 & -0.06100 & -2.06294 \\ 7 & -1.85854 & -0.02704 & -3.25912 \\ 1 & 0.53255 & 0.75676 & -2.89017 \\ 1 & -2.75300 & -0.49750 & -3.25124 \\ 1 & -2.38784 & -1.11477 & 1.66342 \\ 9 & 3.24422 & 0.92260 & 1.18661 \\ 1 & 1.52610 & 0.19066 & 1.95524\end{array}$

8oxoguanine with hydrogen fluoride at $\mathrm{O} 8(\mathrm{~N} 7)$

$\begin{array}{llll}6 & -0.18075 & -0.45618 & 2.00690 \\ 8 & -0.15964 & -0.69280 & 3.22419 \\ 7 & -1.27476 & -0.61074 & 1.16027 \\ 7 & 0.83880 & 0.00312 & 1.21275 \\ 1 & 1.29593 & -0.36892 & 3.74422 \\ 6 & 0.39069 & 0.13223 & -0.10233 \\ 6 & -0.93626 & -0.25292 & -0.12906 \\ 1 & -1.47432 & 0.10102 & -4.35801 \\ 6 & 1.03985 & 0.56908 & -1.29094 \\ 7 & -1.76009 & -0.29250 & -1.19941 \\ 8 & 2.19057 & 0.95376 & -1.46795 \\ 7 & 0.11712 & 0.51766 & -2.38604 \\ 6 & -1.18967 & 0.11102 & -2.31630 \\ 7 & -1.93569 & 0.18043 & -3.46353 \\ 1 & 0.49935 & 0.87137 & -3.25590 \\ 1 & -2.84194 & -0.26236 & -3.40101 \\ 1 & 1.76256 & 0.20290 & 1.58037 \\ 9 & 2.22735 & -0.09986 & 3.76514 \\ 1 & -2.17598 & -0.93803 & 1.47624\end{array}$

8oxoguanine with hydrogen fluoride at N3(N2)

$\begin{array}{llll}6 & -0.03813 & 0.55730 & 2.62280 \\ 8 & 0.11607 & 0.90424 & 3.78019 \\ 7 & 0.84292 & 0.79567 & 1.54221 \\ 7 & -1.08696 & -0.14699 & 2.05308 \\ 1 & 2.24887 & 1.01335 & -1.40242 \\ 6 & -0.86494 & -0.33496 & 0.68886 \\ 6 & 0.34413 & 0.25778 & 0.38302 \\ 1 & 0.18791 & -0.54783 & -3.85500 \\ 6 & -1.63230 & -0.98507 & -0.31268 \\ 7 & 0.92607 & 0.30563 & -0.84117 \\ 8 & -2.71184 & -1.56231 & -0.23023 \\ 7 & -0.95553 & -0.90387 & -1.57759 \\ 6 & 0.24462 & -0.29695 & -1.80960 \\ 7 & 0.77207 & -0.34260 & -3.05986 \\ 1 & -1.43609 & -1.38840 & -2.32721 \\ 1 & 1.61241 & 0.20469 & -3.21948 \\ 1 & -1.88565 & -0.46797 & 2.57865 \\ 9 & 2.94622 & 1.33452 & -2.00884 \\ 1 & 1.71246 & 1.29647 & 1.65418\end{array}$




\begin{tabular}{llll}
\multicolumn{4}{l}{ 8oxoguanine with hydrogen fluoride at O6(N1) } \\
6 & -0.56464 & -0.85878 & 2.69445 \\
8 & -0.70391 & -1.18083 & 3.86071 \\
7 & -1.48604 & -1.06306 & 1.64258 \\
7 & 0.50644 & -0.21948 & 2.08866 \\
1 & 2.56725 & 1.56344 & -1.60124 \\
6 & 0.25658 & -0.03695 & 0.72906 \\
6 & -0.99824 & -0.57093 & 0.45904 \\
1 & -0.88164 & 0.14854 & -3.77009 \\
6 & 1.00460 & 0.54306 & -0.30810 \\
7 & -1.61903 & -0.61074 & -0.73483 \\
8 & 2.14417 & 1.06450 & -0.24488 \\
7 & 0.31465 & 0.49094 & -1.53986 \\
6 & -0.92463 & -0.06049 & -1.71578 \\
7 & -1.47135 & 0.00684 & -2.96384 \\
1 & 0.81701 & 0.92864 & -2.31559 \\
1 & -2.31156 & -0.53517 & -3.10287 \\
1 & 1.33388 & 0.06385 & 2.59078 \\
9 & 2.55301 & 1.77199 & -2.56772 \\
1 & -2.37877 & -1.51348 & 1.78091
\end{tabular}

\begin{tabular}{llll}
\multicolumn{4}{l}{ 8oxoguanine with water at O6(N7) } \\
6 & -0.41378 & -0.04568 & 2.38137 \\
8 & -0.44224 & -0.06865 & 3.60062 \\
7 & -1.53881 & -0.03024 & 1.51895 \\
7 & 0.67291 & -0.03144 & 1.52790 \\
8 & 3.48301 & 0.08456 & 1.19152 \\
6 & 0.24263 & -0.00368 & 0.20303 \\
6 & -1.14619 & -0.00492 & 0.20706 \\
1 & -1.65140 & 0.43099 & -4.01530 \\
6 & 0.94464 & 0.00705 & -1.02439 \\
7 & -1.96463 & 0.02636 & -0.86872 \\
8 & 2.16599 & -0.00378 & -1.25308 \\
7 & 0.03758 & 0.02676 & -2.11998 \\
6 & -1.32872 & 0.03613 & -2.02059 \\
7 & -2.04689 & 0.00080 & -3.19122 \\
1 & 0.48790 & -0.02583 & -3.02656 \\
1 & -3.03820 & 0.15513 & -3.06636 \\
1 & -2.48807 & -0.03591 & 1.86118 \\
1 & 4.22385 & -0.51565 & 1.33211 \\
1 & 1.65389 & -0.02214 & 1.81536 \\
1 & 3.24529 & 0.03719 & 0.23978
\end{tabular}

\begin{tabular}{llll}
\multicolumn{5}{l}{ 8oxoguanine with water at O8(N7) } \\
6 & -0.26681 & -0.07972 & 2.08023 \\
8 & -0.27044 & -0.13237 & 3.31215 \\
7 & -1.39133 & 0.02356 & 1.25050 \\
7 & 0.80983 & -0.10779 & 1.22318 \\
8 & 2.51422 & -0.18126 & 3.48833
\end{tabular}




$\begin{array}{llll}6 & 0.36999 & -0.01838 & -0.09630 \\ 6 & -1.01096 & 0.06170 & -0.07279 \\ 1 & -1.55521 & 0.62801 & -4.27294 \\ 6 & 1.06957 & -0.02025 & -1.33457 \\ 7 & -1.84701 & 0.16927 & -1.13148 \\ 8 & 2.27239 & -0.10017 & -1.56714 \\ 7 & 0.13384 & 0.08461 & -2.41565 \\ 6 & -1.22744 & 0.17094 & -2.29258 \\ 7 & -1.96558 & 0.20853 & -3.45079 \\ 1 & 0.56390 & 0.02781 & -3.33183 \\ 1 & -2.94320 & 0.42165 & -3.30684 \\ 1 & 1.58380 & -0.19907 & 3.79328 \\ 1 & 3.00821 & -0.79942 & 4.03845 \\ 1 & 1.76380 & -0.16898 & 1.57381 \\ 1 & -2.33503 & 0.06735 & 1.60512\end{array}$

8oxoguanine with water at $\mathrm{N} 3(\mathrm{~N} 2)$

$\begin{array}{llll}6 & 0.41081 & -0.15043 & 2.68902 \\ 8 & 0.39089 & -0.26546 & 3.90346 \\ 7 & -0.69196 & -0.24536 & 1.81232 \\ 7 & 1.48584 & 0.09856 & 1.85284 \\ 8 & -3.65658 & -0.74631 & -1.68790 \\ 6 & 1.06694 & 0.15125 & 0.52213 \\ 6 & -0.29813 & -0.06467 & 0.50672 \\ 1 & -0.70899 & 0.00356 & -3.76850 \\ 6 & 1.77822 & 0.37529 & -0.68442 \\ 7 & -1.10494 & -0.10114 & -0.58023 \\ 8 & 2.97469 & 0.58799 & -0.86870 \\ 7 & 0.87459 & 0.33021 & -1.79489 \\ 6 & -0.47543 & 0.10692 & -1.73012 \\ 7 & -1.18455 & 0.14233 & -2.89032 \\ 1 & 1.31606 & 0.52547 & -2.68612 \\ 1 & -2.15910 & -0.15420 & -2.83204 \\ 1 & -4.46932 & -0.28406 & -1.45129 \\ 1 & -3.00118 & -0.56388 & -0.98369 \\ 1 & 2.42957 & 0.21768 & 2.18747 \\ 1 & -1.62627 & -0.43673 & 2.14123\end{array}$

\begin{tabular}{llll}
\multicolumn{4}{l}{ 8oxoguanine with water at O6(N1) } \\
6 & -0.22294 & -0.53704 & 2.86103 \\
8 & -0.20955 & -0.74529 & 4.06302 \\
7 & -1.34361 & -0.58278 & 2.00516 \\
7 & 0.83048 & -0.20625 & 2.02343 \\
8 & 2.20282 & 0.92322 & -3.38153 \\
6 & 0.38003 & -0.05431 & 0.71142 \\
6 & -0.98548 & -0.29327 & 0.70993 \\
1 & -1.48738 & 0.05100 & -3.52523 \\
6 & 1.04635 & 0.26748 & -0.49129 \\
7 & -1.81235 & -0.26440 & -0.35541 \\
8 & 2.25478 & 0.50389 & -0.67149
\end{tabular}




$\begin{array}{llll}7 & 0.13393 & 0.30505 & -1.57828 \\ 6 & -1.20400 & 0.04605 & -1.48623 \\ 7 & -1.94474 & 0.16403 & -2.63223 \\ 1 & 0.55961 & 0.58104 & -2.47014 \\ 1 & -2.88608 & -0.19627 & -2.56774 \\ 1 & 2.62132 & 1.68774 & -3.79405 \\ 1 & 2.57084 & 0.84124 & -2.47246 \\ 1 & 1.77921 & -0.09883 & 2.34734 \\ 1 & -2.27166 & -0.80344 & 2.33436\end{array}$

\begin{tabular}{llll}
\multicolumn{4}{l}{ 8oxoguanine with ammonia at O6(N7) } \\
6 & 0.44493 & -0.62071 & 2.25198 \\
8 & 0.54849 & -0.80501 & 3.45556 \\
7 & 1.12883 & -1.33795 & 1.23846 \\
7 & -0.32917 & 0.29446 & 1.56728 \\
7 & -2.23824 & 2.40541 & 1.99158 \\
6 & -0.13476 & 0.15038 & 0.19549 \\
6 & 0.77868 & -0.87474 & -0.00352 \\
1 & 1.09930 & -0.44519 & -4.24079 \\
6 & -0.69361 & 0.83116 & -0.91465 \\
7 & 1.25315 & -1.32957 & -1.18722 \\
8 & -1.51906 & 1.75220 & -0.95821 \\
7 & -0.16957 & 0.30037 & -2.13271 \\
6 & 0.74391 & -0.71533 & -2.23248 \\
7 & 1.08949 & -1.13087 & -3.49855 \\
1 & -0.58357 & 0.70232 & -2.96598 \\
1 & 1.86956 & -1.77414 & -3.51219 \\
1 & -0.96852 & 0.98901 & 1.99241 \\
1 & -1.95980 & 3.30290 & 2.37896 \\
1 & -3.17816 & 2.20641 & 2.32331 \\
1 & -2.26687 & 2.48871 & 0.97290 \\
1 & 1.77633 & -2.08505 & 1.43982
\end{tabular}

\begin{tabular}{llll}
\multicolumn{4}{l}{ 8oxoguanine with ammonia at O8(N7) } \\
6 & -0.41196 & 0.60772 & 2.01111 \\
8 & -0.53561 & 0.82727 & 3.21394 \\
7 & -1.04583 & 1.30540 & 0.96460 \\
7 & 0.35551 & -0.35067 & 1.38544 \\
7 & 1.50417 & -1.58485 & 3.70313 \\
6 & 0.20521 & -0.25315 & 0.00454 \\
6 & -0.67249 & 0.78692 & -0.25330 \\
1 & -0.89682 & 0.23272 & -4.48102 \\
6 & 0.77804 & -0.98951 & -1.06815 \\
7 & -1.10559 & 1.21810 & -1.46219 \\
8 & 1.57360 & -1.92650 & -1.05651 \\
7 & 0.29478 & -0.47865 & -2.31819 \\
6 & -0.58680 & 0.55696 & -2.47369 \\
7 & -0.88972 & 0.94107 & -3.76045 \\
1 & 0.71831 & -0.91803 & -3.12741 \\
1 & -1.65220 & 1.60291 & -3.81498
\end{tabular}




$\begin{array}{llll}1 & 0.92780 & -1.00334 & 1.94170 \\ 1 & 2.46973 & -1.44202 & 3.98677 \\ 1 & 1.21114 & -2.49229 & 4.05500 \\ 1 & 0.93081 & -0.86868 & 4.15122 \\ 1 & -1.67801 & 2.07613 & 1.11980\end{array}$

8oxoguanine with ammonia at N3(N2)

$\begin{array}{llll}6 & -0.02160 & 0.14113 & 2.77178 \\ 8 & -0.15246 & 0.38513 & 3.96145 \\ 7 & -0.50303 & 0.91096 & 1.69291 \\ 7 & 0.61616 & -0.92762 & 2.16642 \\ 7 & -1.99575 & 2.82316 & -2.80288 \\ 6 & 0.53342 & -0.82090 & 0.77566 \\ 6 & -0.16905 & 0.33503 & 0.48676 \\ 1 & -0.12341 & -0.17513 & -3.77491 \\ 6 & 1.02273 & -1.64622 & -0.26769 \\ 7 & -0.48446 & 0.82094 & -0.73432 \\ 8 & 1.66427 & -2.69523 & -0.21206 \\ 7 & 0.66639 & -1.08540 & -1.53441 \\ 6 & -0.03831 & 0.07623 & -1.73775 \\ 7 & -0.24853 & 0.47757 & -3.01716 \\ 1 & 1.02209 & -1.61044 & -2.32477 \\ 1 & -0.88668 & 1.27375 & -3.15812 \\ 1 & 1.07130 & -1.66380 & 2.68341 \\ 1 & -1.81111 & 2.68708 & -1.80915 \\ 1 & -3.00233 & 2.80049 & -2.94323 \\ 1 & -1.66851 & 3.75012 & -3.06187 \\ 1 & -1.01459 & 1.76978 & 1.82701\end{array}$

\begin{tabular}{llll}
\multicolumn{4}{l}{ 8oxoguanine with ammonia at O6(N1) } \\
6 & -0.78127 & 1.04143 & 2.65695 \\
8 & -1.05475 & 1.39430 & 3.79383 \\
7 & -1.14299 & 1.68925 & 1.45868 \\
7 & -0.05900 & -0.06460 & 2.24012 \\
7 & 1.88072 & -2.71346 & -2.72710 \\
6 & 0.02271 & -0.09724 & 0.84643 \\
6 & -0.65967 & 1.00693 & 0.36498 \\
1 & -0.17531 & 0.03999 & -3.77501 \\
6 & 0.63682 & -1.00632 & -0.04951 \\
7 & -0.83286 & 1.35442 & -0.92806 \\
8 & 1.27951 & -2.03574 & 0.20411 \\
7 & 0.43367 & -0.59430 & -1.39478 \\
6 & -0.25749 & 0.51868 & -1.77483 \\
7 & -0.30935 & 0.79413 & -3.11774 \\
1 & 0.88016 & -1.21351 & -2.09586 \\
1 & -0.97931 & 1.50220 & -3.38125 \\
1 & 0.34181 & -0.73385 & 2.87868 \\
1 & 1.97176 & -2.94093 & -1.73329 \\
1 & 2.81630 & -2.58621 & -3.10513 \\
1 & 1.47013 & -3.51735 & -3.19551
\end{tabular}


1.44178 